In all the foregoing instances we have a successful correlation of the results of physical chemistry with those obtained by the pure chemist in the deduction of constitutional formulæ. Many who withheld judgment, or even condemned, when the chemist was his own witness, may listen to him more favourably now that he is supported by the independent testimony of the physicist.

These investigations into the physical properties of organic compounds are of relatively recent date. There is little doubt that as they are extended new and important laws will be deduced. Much is to be hoped from thermo-chemistry, incoherent as many of its utterances as yet are. By following the path of physico-chemical research, chemists may even hope to arrive at a dynamical representation of the molecule which shall be as much more powerful as an instrument of research than the present merely statical constitutional formula, as that is more powerful than the empirical formula which preceded it. It is hazardous to try to fix a limit to scientific advance in any direction, but it is probable that the modern constitutional formula represents the limit to what purely chemical research can accomplish in determining the constitution of matter. Much will still have to be done by purely chemical research in working out the details of the existing system: the constitution of the more complex compounds will be ascertained on the lines of our present formulæ; new valuable natural compounds will be synthesized. But mere chenical reactions can probably never settle questions of intramolecular dynamics; in these help must come from physical chemistry. Moreover, the physical methods of research supplement the chemical methods in one important particular. By chemical methods we can never study the molecule as it actually exists. Uur synthetical methods give us information concerning the molecule only at the moment of its formation; our analytical methods equally confine themselves to the monent of its destruction. The physical methods supply this want: they enable us to study the existing compound. Of these physical methods, one of the most promising, although one of the most recent, is the optical method, which has yielded results of the utmost importance both in inorganic and in organic chemistry. The ray of light which passes from the fixed star to the earth gives us information concerning the composition of the atmosphere of the fixed star; and it is perhaps not too much to hope that the ray of light which has threaded its way through and between the molecules of a compound, and has been modified by its contact with these, will, if properly interrogated furnish some information concerning the structure of these molecules. Indeed, in the case of the rotation of the plane of polarized light by organic liquids, of their absorption spectra and their indices of refraction, this information has in a measure been obtained.

To sum up. The constitutional formula is not an ultimate expression of the whole truth as regards molecular structure. But it is certainly a very useful and convenient symbolical expression of certain aspects of the truth. We all hope that it may one day be superseded by some higher and more complete generalization. But it will be absorbed and assimilated, not rejected and contradicted, by that generalization. Non ommis morietur.

\section{THE YALE COLLEGE MEASUREMENT OF} THE PLEIADES.1

THE Messrs. Repsold have established, and for the present seem likely to maintain, a practical monopoly in the construction of heliometers. That completed by them for the Observatory of Yale College in

x "Determination of the Relative Positions of the Principal Stars in the Group of the Pleiades." By William L. Elkin. Transactions of the Astronomical Observatory of Yale University. Vol. I., Part I. (New Haven: 1887.)
I 882 leaves so little to be desired as to show excellence not to be the exclusive result of competition. In mere size it does not indeed take the highest rank ; its aperture is of only 6 inches, while that of the Oxford heliometer is of $7 \frac{1}{2}$; but the perfection of the arrangements adapting it to the twofold function of equatorial and micrometer, stamps it as a model not easy to be surpassed. Steel has been almost exclusively used in the mounting. Recommended as the material for the objectivecell by its quality of changing volume under variations of temperature nearly pari passu with glass, its employment was extended to the telescope-tube and other portions of the mechanism. The optical part of the work was done by Merz, Alvan Clark having declined the responsibility of dividing the object-lens. Its segments are separable to the extent of $2^{\circ}$, and through the contrivance of cylindrical slides (originally suggested by Bessel) perfect definition is preserved in all positions, giving a range of accurate measurement just six times that with a filar micrometer. (Gill, "Encyc. Brit." vol. xvi, p. 253 ; Fischer, Sirius, vol. xvii. p. I45.)

This beautiful engine of research was in 1883 placed in the already practised and skilful hands of Dr. Elkin. He lost no time in fixing upon a task suited both to test the powers of the new instrument and to employ them to the highest advantage.

The stars of the Pleiades have, from the earliest times, attracted the special notice of observers, whether savage or civilized. Hence, on the one hand, their prominence in stellar mythology all over the world; on the other, their unique interest for purposes of scientific study and comparison. They constitute an undoubted cluster; that is to say, they are really, and not simply in appearance, grouped together in space, so as to fall under the sway of prevailing mutual influences. And since there is, perhaps, no other stellar cluster so near the sun, the chance of perceptible displacements among them in a moderate lapse of time is greater than in any other similar case. Authentic data regarding them, besides, have now been so long garnered that their fruit may confidently be expected at least to begin to ripen.

Dr. Elkin determined, accordingly, to repeat the survey of the Pleiades executed by Bessel at Königsberg during about twelve years previous to I84I. Wolf and Pritchard had, it is true, been beforehand with him; but the wide scattering of the grouped stars puts the filar micrometer at a disadvantage in measuring them, producing minute errors which the arduous conditions of the problem render of serious account. The heliometer, there can be no doubt, is the special instrument for the purpose, and it was, moreover, that employed by Bessel; so that the Königsberg and Yale results are comparable in a stricter sense than any others so far obtained

One of Besiel's fifty-three stars was omitted by Dr. Elkin as too faint for accurate determination. He added, however, seventeen stars from the Bonn Durchmusterung, so that his list comprised sixty-nine, down to $9^{\prime} 2$ magnitude. Two independent triangulations were executed by him in 1884-85. For the first, four stars situated near the outskirts of the group, and marking the angles of a quadrilateral by which it was inclosed, were chosen as reference-points. The second rested upon measures of distance and position-angle outward from Alcyone ( $\eta$ Tauri). Thus, two wholly unconnected sets of positions were secured, the close accordance of which testified strongly to the high quality of the entire work. They were combined, with nearly equal weights, in the final results. A fresh reduction of the Königsberg observations, necessitated by recent improvements in the value of some of the corrections employed, was the preliminary to their comparison with those made, after an interval of forty-five years, at Yale College. The conclusions thus laboriously arrived at are not devoid of significance, and appear perfectly secure, so far as they go. 
It has been known for some time that the stars of the Pleiades possess a small identical proper motion. Its direction, as ascertained by Newcomb in 1878 , is about south-south-east; its amount is somewhat less than six seconds of arc in a century. The double-star 6I Cygni, in fact, is displaced very nearly as much in one year as Alcyone with its train in one hundred. Nor is there much probability that this slow secular shifting is other than apparent: since it pretty accurately reverses the course of the sun's translation through space, it may be presumed that the backwari current of movement in which the Pleiades seem to float is purely an effect of our own onward travelling.

Now the curious fact emerges from Dr. Elkin's inquiries that six of Bessel's stars are exempt from the general drift of the group. They are being progressively left behind. The inference is obvious, that they do not in reality belong to, but are merely accidentally projected upon, it: or, rather, that it is projected upon them; for their apparent immobility (which, in two of the six, may be called absolute) shows them with tolerable certainty to be indefinitely more remoteso remote that the path, moderately estimated at $21,000,000,000$ miles in length, traversed by the solar system during the forty-five years elapsed since the Königsberg measures, dwindles into visual insensibility when beheld from them ! The brightest of these six far-of stars is just above the eighth $\left(7^{\circ} 9\right)$ magnitude; the others range from 8.5 down to below the ninth.

A chart of the relative displacements indicated for Bessel's stars by the differences in their inter-mutual positions as determined at Königsberg and Yale, accompanies the paper before us. Divergences exceeding o"40 (taken as the limit of probable error) are regarded as due to real motion; and this is the case with twenty-six stars besides the half-dozen already mentioned as destined deserters from the group. With these last may be associated two stars surmised, for an opposite reason, to stand aloof from it. Instead of tarrying behind, they are hurrying on in front. An excess of the proper movement of their companions belongs to them; and since that movement is presumably an effect of secular parallax, we are justified in inferring their possession of an extra share of it to signify their greater proximity to the sun. Hence, of all the stars in the Pleiades these are the most likely to have a measurable annual parallax. One is a star a little above the seventh magnitude, distinguished as $s$ Pleiadum; the other, of about the eighth, is numbered 25 in Bessel's list. Dr. Elkin has not omitted to remark that the conjecture of their disconnexion from the cluster is confirmed by the circumstance that its typical spectrum (as shown on Prof. Pickering's plates) is varied in $s$ by the marked character of the $\mathrm{K}$ line. The spectrum of its fellowtraveller (No. 25) is still undetermined.

It is improbable, however, that even these nearer stars are practicable subjects for the direct determination of annual parallax. By indirect means, however, we can obtain some idea of their distance. All that we want to know for the purpose is the rate of the sun's motion; its direction we may consider as given with approximate accuracy by Airy's investigation. Now, spectroscopic measurements of stellar movements of approach and recession will eventually afford ample materials from which to deduce the solar velocity; though they are as yet not accurate or numerous enough to found any definitive conclusion upon. Nevertheless, M. Homann's preliminary result of fifteen miles a second as the speed with which our system travels in its vast orbit, inspires confidence both from the trustworthiness of the determinations (Mr. Seabroke's) serving as its basis, and from its intrinsic probability. Accepting it provisionally, we find the parallax of Alcyone = about $\mathrm{O}^{\prime \prime} \cdot \mathrm{O} 2$, implying a distance of $954,000,000,000,000$ miles, and a light-journey of 163 years. Jt is assumed that the whole of its proper motion of $2^{\prime \prime} \cdot 6 \mathrm{I}$ in forty-five years is the visual projection of our own movement towards a point in R.A. $261^{\circ}$, Decl. $+25^{\circ}$.

Thus, the parallax of the two stars which we suspect to lie between us and the stars forming the genuine group of the Pleiades, at perhaps two-thirds of their distance, can hardly exceed $o^{\prime \prime} \cdot 03$. This is just half that found by Dr. Gill for $\zeta$ Toucani, which may be regarded as, up to this, the smallest annual displacement at all satisfactorily determined. And the error of the present estimate is more likely to be on the side of excess than of defect. That is, the stars in question can hardly be much nearer to us than is implied by an annual parallax of $\mathrm{O}^{\prime \prime} \cdot 03$, and they may be considerably more remote.

Dr. Elkin concludes, from the minuteness of the detected changes of position among the Pleiades, that " the hopes of obtaining any clue to the internal mechanism of this cluster seem not likely to be realized in an immediate future;" remarking further: "The bright stars in especial seem to form an almost rigid system, as for only one is there really much evidence of motion, and in this case the total amount is barely I" per century." This one mobile member of the naked-eye group is Electra ; and it is noticeable that the apparent direction of its displacement favours the hypothesis of leisurely orbital circulation round the leading star. The larger movements, however, ascribed to some of the fainter associated stars are far from harmonizing with this preconceived notion of what they ought to be. On the contrary, so far as they are known at present, they force upon our minds the idea that the cluster may be undergoing some slow process of disintegration. M. Wolf's impression of incipient centrifugal tendencies among its components certainly derives some confirmation from Dr. Elkin's chart. Divergent movements are the most strongly marked; and the region round Alcyone suggests, at the first glance, rather a very confused area of radiation for a flight of meteors, than the central seat of attraction of a revolving throng of suns.

There are many signs, however, that adjacent stars in the cluster do not pursue independent courses. "Community of drift" is visible in many distinct sets; while there is as yet no perceptible evidence, from orbital motion, of association into subordinate systems. The three eighth-magnitude stars, for instance, arranged in a small isosceles triangle near Alcyone, do not, as might have been expected a priori, constitute a real ternary group. They are all apparently travelling directly away from the large star close by them, in straight lines which may of course be the projections of closed curves; but their rates of travel are so different as to involve certain progressive separation. Obviously, the order and method of such movements as are just beginning to develop to our apprehension among the Pleiades will not prove easy to divine.

A. M. CleRKE.

\section{NOTES.}

STRENUOUS efforts have been made to secure that the arrangements for the observation of the total solar eclipse of August r9 snall be adequate. "A large number of astronomers," says the Times of the $15^{\text {th }}$ inst., "will be distributed along the central line, fully equipped with instruments suited to the particular work they intend to do. The Russians themselves have most energetically organized a very complete set of observations, meteorological and otherwise, at widely-distant stations, viz. Krasnoiarsk in Siberia, Perm in the Ural Mountains, and Viatka in Central Russia; while Prof. Mendeljew goes to Pavlovsk, near St. Petersburg; Prof. Bredichin, of the Moscow Observatory, to Kineshma; and Dr. Podsolnotschnaja will be stationed near Tver. Several foreign astronomers will also visit Russia, and have received very hospitable treatment at the hands of Prof. Struve and the other Russian authorities. From 